\title{
Regression of eosinophil counts after diagnosis of chronic graft-versus-host disease as a potential marker for improved clinical outcome
}

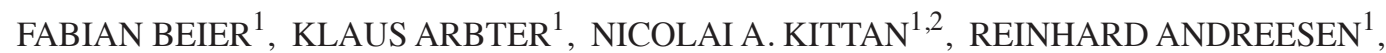 \\ STEFAN W. KRAUSE ${ }^{1,3}$, ERNST HOLLER ${ }^{1}$ and GERHARD C. HILDEBRANDT ${ }^{1,2}$ \\ ${ }^{1}$ Department of Hematology and Oncology, University of Regensburg Medical Center, D-93053 Regensburg, Germany; \\ ${ }^{2}$ Feist-Weiller Cancer Center, Department of Medicine, Louisiana State University Health Sciences Center, Shreveport, \\ LA 71130, USA; ${ }^{3}$ Department of Hematology and Oncology, University of Erlangen Medical Center, \\ D-91054 Erlangen, Germany
}

Received October 9, 2012; Accepted April 16, 2013

DOI: $10.3892 / \mathrm{mco} .2013 .192$

\begin{abstract}
Eosinophilia after allogeneic hematopoietic stem cell transplantation (allo-HSCT) has been associated with the development of acute and chronic graft-versus-host disease (cGVHD). However, a limited number of studies have investigated the course of eosinophil counts in relation to the onset of cGVHD. In this study, the course of relative eosinophil counts (RECs) was retrospectively analyzed in 64 patients who developed cGVHD following allogeneic HSCT in relation to overall survival (OS), relapse rate and clinical course of cGVHD. At onset of cGVHD, eosinophilia was observed in $45 \%$ of the patients and developed one week prior to cGVHD diagnosis. Furthermore, a trend towards improved OS in patients with eosinophilia was observed. Beneficial effects were most evident in patients who exhibited decreasing eosinophil counts one week after diagnosis of cGVHD. By contrast, an increase in or stable eosinophil counts one week after diagnosis were associated with significantly impaired OS and a significantly higher rate of later aggravation of cGVHD. Findings of this study suggested that the course of eosinophil counts may provide a useful parameter in the assessment of cGVHD development and activity allowing the potential identification of patient subpopulations with a good outcome and reduced cGVHD-related mortality.
\end{abstract}

\section{Introduction}

Although eosinophils constitute only $1-4 \%$ of the peripheral blood leukocytes, they may be important in various inflammatory infectious and allergic diseases (1). Chronic

Correspondence to: Dr Gerhard Hildebrandt, Feist-Weiller Cancer Center, Louisiana State University Health Sciences Center, 1501 Kings Highway, Shreveport, LA 71130, USA

E-mail: ghilde@1suhsc.edu

Key words: allogeneic stem cell transplantation, eosinophilia, graft-versus-host disease, $\mathrm{T}$ helper 2 cell graft-versus-host disease (cGVHD) is a serious complication of allogeneic hematopoietic stem cell transplantation (allo-HSCT). cGVHD can occur in almost every organ and clinical diagnosis and staging are challenging (reviewed in NIH cGVHD consensus conference guidelines) $(2,3)$. Although the pathophysiology of cGVHD remains ambiguous, previous data have shown the involvement of a T helper (Th) 2 cell-mediated process with overexpression of Th2 cytokines, such as IL-4 and IL-5 (4-6), and the correlation of eosinophilia with the development of acute and cGVHD (7-17). Th2-mediated immune responses linked to eosinophilia are regularly identified in allograft rejection following solid organ transplantation $(18,19)$. Investigations of the course of eosinophilia suggest a role of eosinophil counts as a predictive marker for graft rejection in these patients (19).

The majority of studies investigating the role of eosinophils in allo-HSCT have focused on the incidence of eosinophilia in relation to overall survival (OS) and relapse-related mortality in patients developing acute $\operatorname{GVHD}(8,10,11,14,15,17)$. A limited number of studies are available regarding the development of chronic GHVD, and their focus is on eosinophilia as a risk factor rather than on the course of peripheral blood eosinophils in the context of time of diagnosis and initial treatment of cGVHD $(9,12-14,16)$.

The aim of this study was to investigate the role of eosinophil counts in cGVHD. Our findings suggest that eosinophil count changes constitute a possible indicator for overall cGVHD activity, severity and treatment response, aiding clinicians in the diagnosis and management of cGVHD patients.

\section{Patients and methods}

Patients. The clinical records of 64 patients developing cGVHD after allo-HSCT at the University of Regensburg Medical Center (Regensburg, Germany) between June, 1998 and December, 2003 were included in this study and retrospectively analyzed. The retrospective analysis for this study was approved by the ethics committee of the 
University of Regensburg (Germany). Regular follow-up was performed at least two years after allo-HSCT (median, 66.7 months).

The patients received standard infectious prophylaxis with aciclovir, fluconazole, co-trimoxazole and ciprofloxacin alone or in combination with metronidazole. Intravenous immunoglobulins were administered in case of severe hypogammaglobulinemia $(<4 \mathrm{~g} / \mathrm{l})$. Patient characteristics are provided in Table I.

Acute GVHD was treated with prednisolone and other immunosuppressive agents as needed. Cyclosporin A (CSA) was usually discontinued 6 months after HSCT if there was no evidence of GVHD. Acute and cGVHD were initially diagnosed and retrospectively reassessed according to the respective official diagnosis criteria $(3,16)$. Among the 64 patients developing cGVHD, localized mucosal/skin involvement or hepatic dysfunction due to cGVHD was classified as limited cGVHD in 16 patients (25\%) and as extensive cGVHD in 48 patients (75\%). Initial first-line therapy for cGVHD was prednisolone (1-2 $\mathrm{mg} / \mathrm{kg}$ body weight) or topic steroids. Fifty patients $(78 \%)$ additionally received systemic/topic CSA or CSA dose escalation at diagnosis of cGVHD. Twenty-one patients (33\%) exhibited later clinical cGVHD aggravation during follow-up defined by further intensification of steroid treatment.

Methods. Eosinophil counts were retrospectively evaluated on days $-14,-7,0$ and +7 in relation to the first day of cGVHD diagnosis with an allowed deviation of 2 days to the date of the blood samples on days -14 and -7 . Eosinophilia was defined as a peripheral relative eosinophil count (REC) of $>4 \%$ according to the value used in recent publications and the internal reference standards of our Institution $(2,8,19)$. In addition, clinically relevant changes in eosinophil counts were assumed in an increase/decrease of at least $1 \%$. For further analysis, patients were stratified into three groups based on the course of eosinophil counts 7 days after cGVHD diagnosis compared to eosinophil counts at cGVHD diagnosis. The first group (decreasing eosinophils, $\mathrm{n}=25$ ) showed decreasing eosinophil counts after cGVHD diagnosis of $\geq 1 \%$. The second group (intermediate eosinophils, $n=30$ ) comprised patients with intermediate eosinophil counts, which were not increasing and not decreasing $>1 \%$ at day +7 . Compared to group 1 , the patients of the third group (increasing eosinophils, $n=9$ ) exhibited increasing RECs of $\geq 1 \% 7$ days after cGVHD diagnosis.

Statistical analysis. Statistical analysis was performed using SPSS version 17. Univariate analysis was performed using the $\chi^{2}$ test, Fisher's exact test and paired Student's t-test as indicated. Multivariate analysis was performed by Cox's proportional hazard analysis. For OS, actuarial curves were obtained using the Kaplan-Meier method and were statistically compared using the log-rank test. In all analyses, a two-sided significance level of $\mathrm{P}=0.05$ was considered to indicate a statistically significant difference.

\section{Results}

$O S, R R$ and $c G V H D$ aggravation. Previous studies suggested eosinophilia as an indicative parameter for clinical outcome $(8,11,14)$. Therefore, we analyzed the OS, relapse rate (RR) and cGVHD aggravation of the patients included in this study. A trend towards improved OS was observed in patients with eosinophilia compared to those without eosinophilia ( $\mathrm{P}=0.11$, Fig. 1). No difference was observed between the two patient groups with regard to relapse rate $(\mathrm{P}=0.29)$, limited cGVHD $(\mathrm{P}=0.10)$ and later cGVHD aggravation $(\mathrm{P}=0.60)$.

Course of peripheral blood eosinophils in cGVHD patients. Since RECs potentially reflect the activity of cGVHD, we investigated patients based on the development of the eosinophil course one week after cGVHD diagnosis. The analysis of the course of RECs demonstrated that patients with a decrease of $>1 \%$ the week after diagnosis, exhibited a significant increase of RECs the week prior to cGVHD diagnosis (Fig. 2A). In patients with an additional increase of eosinophil counts the week following cGVHD diagnosis, a significant increase of eosinophils to the same levels as in the decreasing course of RECs group was observed (Fig. 2B). Notably, the analysis of patients without a significant change of RECs after diagnosis did not demonstrate any significant changes before and after cGVHD diagnosis (Fig. 2C). In agreement with these findings, significantly more patients with eosinophilia were observed in the decreasing $(88 \%, \mathrm{P} \leq 0.001)$ and increasing $(66 \%, \mathrm{P} \leq 0.001)$ groups compared to the intermediate course of RECs group (7\%).

Changes of eosinophil counts after cGVHD diagnosis in the three groups. OS was then analyzed in relation to the eosinophil course after diagnosis. The analysis of the Kaplan-Meier survival curve demonstrated a significantly improved OS $(\mathrm{P}=0.04)$ in the decreasing compared to the increasing course of RECs group (Fig. 3). The survival curve of patients without significant changes of RECs also showed a significantly impaired OS compared to patients with increasing $(\mathrm{P}=0.52)$ and decreasing eosinophil groups $(\mathrm{P}=0.03)$. Focusing on potential factors in explaining the different OS rates, no significant correlation was observed between organ manifestation of cGVHD and eosinophil counts in the three groups ( $P>0.05)$. Regarding the rates of limited cGVHD disease and the overlap of acute and cGVHD, no significant differences were observed among the three groups $(P>0.05$, Table I). Proceeding acute GVHD was found to occur more frequently in the increasing $(100 \%, \mathrm{P}=0.04)$ and intermediate groups (93\%, $\mathrm{P}=0.007)$ compared to the decreasing course of RECs group (58\%). Notably, the analysis of relapse rate did not differ among the three groups ( $\mathrm{P}>0.05)$, while cGVHD aggravation after diagnosis occurred significantly less frequently in patients with decreasing (12\%) compared to patients with an increasing course of RECs $(66 \%, \mathrm{P}=0.004)$. A strong tendency towards a higher incidence of later $\mathrm{CGVHD}$ aggravation was observed in patients with decreasing compared to patients with no significant changes in the course of RECs $(37 \%, \mathrm{P}=0.06)$.

Steroid treatment. The role of steroid treatment in the three groups was also analyzed, possibly affecting the eosinophil course after cGVHD diagnosis. The percentage of patients administered steroid dose escalation (1-2 $\mathrm{mg} / \mathrm{kg}$ body weight) did not differ between patients with decreasing (60\%) and increasing RECs (44\%, $\mathrm{P}=0.46)$. 
Table I. Characteristics of the three cGVHD patient groups $(n=64)$ based on the course of eosinophil counts seven days after cGVHD diagnosis.

\begin{tabular}{|c|c|c|c|}
\hline \multirow[b]{2}{*}{ Patient characteristics } & \multicolumn{3}{|c|}{ Course of eosinophil counts, $\mathrm{n}(\%)$} \\
\hline & $\begin{array}{l}\text { Decreasing } \\
(\mathrm{n}=26,41 \%)\end{array}$ & $\begin{array}{l}\text { Intermediate } \\
(\mathrm{n}=29,45 \%)\end{array}$ & $\begin{array}{l}\text { Increasing } \\
(\mathrm{n}=9,14 \%)\end{array}$ \\
\hline Age (years) & $48.6 \pm 11.4$ & $42.2 \pm 11.8$ & $38.6 \pm 11.4$ \\
\hline Gender (female/male) & $8(30) / 18(70)$ & $14(48) / 15(52)$ & $3(33) / 6(66)$ \\
\hline \multicolumn{4}{|l|}{ Disease } \\
\hline AML & $9(35)$ & $6(21)$ & $1(11)$ \\
\hline ALL & $0(0)$ & $3(10)$ & $3(33)$ \\
\hline CML & $3(12)$ & $5(17)$ & $0(0)$ \\
\hline NHL & $6(23)$ & $10(35)$ & $5(56)$ \\
\hline CLL & $1(4)$ & $3(10)$ & $0(0)$ \\
\hline MM & $2(8)$ & $1(3)$ & $0(0)$ \\
\hline MF & $4(15)$ & $0(0)$ & $0(0)$ \\
\hline $\mathrm{AA} / \mathrm{PNH}$ & $1(4)$ & $0(0)$ & $0(0)$ \\
\hline Other & $0(0)$ & $1(3)$ & $0(0)$ \\
\hline Sibling/unrelated donor & $15(58) / 11(42)$ & $13(45) / 16(55)$ & $6(66) / 3(33)$ \\
\hline HLA identical/MM & $22(85) / 4(15)$ & $27(93) / 2(7)$ & $8(89) / 1(11)$ \\
\hline \multicolumn{4}{|l|}{ Stem cell source } \\
\hline Bone marrow & $6(23)$ & $5(17)$ & $1(11)$ \\
\hline Selected PBSC & $8(31)$ & $4(14)$ & $0(0)$ \\
\hline Unselected PBSC & $12(46)$ & $20(69)$ & $8(89)$ \\
\hline \multicolumn{4}{|l|}{ Conditioning regimens } \\
\hline TBI-based protocols (>8 Gy) & $10(39)$ & $16(55)$ & $5(56)$ \\
\hline FBM protocol & $9(35)$ & $9(31)$ & $4(44)$ \\
\hline Other protocols & $7(27)$ & $4(14)$ & $0(0)$ \\
\hline \multicolumn{4}{|l|}{ GVHD prophylaxis } \\
\hline ATG $10 \mathrm{mg} / 20 \mathrm{mg}$ & $4(15) / 5(19)$ & $9(31) / 6(21)$ & $2(22) / 2(22)$ \\
\hline CSA alone & $5(19)$ & $3(10)$ & $2(22)$ \\
\hline CSA/MTX & $9(35)$ & $16(55)$ & $5(56)$ \\
\hline $\mathrm{CSA} / \mathrm{MMF}$ & $9(35)$ & $8(28)$ & $1(11)$ \\
\hline Other immunosuppressive drugs & $3(12)$ & $2(7)$ & $1(11)$ \\
\hline Acute GVHD grade II-IV & $15(58)$ & $28(97)$ & $9(100)$ \\
\hline Overlap acute to cGVHD & $5(19)$ & $8(28)$ & $0(0)$ \\
\hline Relapse & $6 / 25(24)$ & $10(35)$ & $3 / 8(38)$ \\
\hline Survival, (months) median & $75.9 \pm 40.0$ & $62.7 \pm 42.0$ & $48.6 \pm 47.2$ \\
\hline Start cGVHD (day) & $215 \pm 89$ & $184 \pm 56$ & $188 \pm 67$ \\
\hline Eosinophilia (>4) at diagnosis & $21(81)$ & $2(7)$ & $6(66)$ \\
\hline cGVHD severity (limited disease) & $5(19)$ & $9(31)$ & $2(22)$ \\
\hline CSA/TAC Tx at cGVHD diagnosis & $22(85)$ & $24(83)$ & $5(55)$ \\
\hline Mean prednisolone dosage on days $-14 /-7$ (mg) & $3.8 / 3.4$ & $18.2 / 14.7$ & $3.1 / 3.3$ \\
\hline Mean prednisolone dosage on days $0 /+7$ (mg) & $50.6 / 44.5$ & $28.7 / 26.3$ & $28.9 / 30.1$ \\
\hline Prednisolone escalation $(1-2 \mathrm{mg} / \mathrm{kg})$ & $16(62)$ & $6(21)$ & $4(44)$ \\
\hline cGHVD aggravation & $3(12)$ & $11(38)$ & $6(66)$ \\
\hline Initiation of cGVHD aggravation (days) & $463 \pm 358$ & $360 \pm 138$ & $373 \pm 241$ \\
\hline
\end{tabular}


Table I. Continued.

Course of eosinophil counts, n (\%)

\begin{tabular}{lccc} 
& & & \\
\cline { 2 - 4 } Patient characteristics & $\begin{array}{c}\text { Decreasing } \\
(\mathrm{n}=26,41 \%)\end{array}$ & $\begin{array}{c}\text { Intermediate } \\
(\mathrm{n}=29,45 \%)\end{array}$ & $\begin{array}{c}\text { Increasing } \\
(\mathrm{n}=9,14 \%)\end{array}$ \\
\hline Organ involvement in cGVHD & & $21(72)$ & $7(78)$ \\
Mucosa & $17(66)$ & $17(59)$ & $4(44)$ \\
Skin & $9(35)$ & $12(41)$ & $2(22)$ \\
Eyes & $8(31)$ & $5(17)$ & $0(0)$ \\
Joints & $2(8)$ & $12(41)$ & $3(33)$ \\
Gastrointestinal & $5(19)$ & $2(7)$ & $3(33)$ \\
Liver & $2(8)$ & $7(24)$ & $2(22)$
\end{tabular}

The absolute number and the respective relative percentage (rounded to integers) are provided. Patient characteristics are reported as the mean \pm standard deviation (SD) and frequencies as a relative percentage. cGVHD, chronic graft-versus-host disease; AML, acute myeloid leukemia; ALL, acute lymphatic leukemia; CML, chronic myeloid leukemia; NHL, non-Hodgkin lymphoma; CLL, chronic lymphatic leukemia; MM, multiple myeloma; MF, marrow fibrosis; AA/PNH, aplastic anemia/paroxysmal nocturnal hemoglobinuria; HLA, human leukocyte antigen; MM, mismatch; TBI, total body irradiation; FBM, fludarabine; ATG, rabbit antithymcoyte globulin (administered over 3 days); CSA, cyclosporin; TAC, tacrolimus; MTX, methotrexate; MMF, mycophenolate mofetil; Tx, treatment. Clinical symptoms for organ affection: mucosa: lichenification, sclerosis and hyperkeratosis; skin: lichenification and sclerosis; eyes: keratokonjunctivitis sicca and all other eye affections; joints: arthritis, arthralgias and sclerosis; gastrointestinal: esophagitis, nausea/vomiting and diarrhea; liver, elevated liver enzymes; lung, obstruction and bronchiolitis obliterans.

Patients without significant changes in the course of RECs exhibited a significantly lower frequency of steroid escalation $(23 \%)$ compared to the decreasing $(\mathrm{P}=0.01)$ group, while this was not the case compared to the increasing course of RECs group $(\mathrm{P}=0.24)$.

Results of multivariate analysis. A multivariate analysis was performed with parameters including age, relapse, previous history of acute GVHD, limited or extended cGVHD and eosinophil behaviour. Age $(\mathrm{P}=0.01)$ and relapse $(\mathrm{P}=0.001)$ were identified as independent factors for OS, in contrast to eosinophil counts $(\mathrm{P}=0.45)$, previous history of acute GVHD $(\mathrm{P}=0.89)$ and limited or extended cGVHD $(\mathrm{P}=0.49)$.

\section{Discussion}

cGVHD significantly contributes to morbidity and mortality after allo-HSCT. Therefore, the identification of risk factors and clinical markers predicting cGVHD and its clinical course are important to enable possible pre-emptive therapy and treatment changes (16).

The role of eosinophilia in cGVHD patients remains ambiguous. Two previous studies have shown a correlation of eosinophilia with improved OS and reduced non-relapse mortality, and have demonstrated no difference in the rate of relapse $(8,14)$. In agreement with findings of those studies, the present study has shown that cGVHD patients with eosinophilia demonstrated a tendency towards an improved outcome in OS, while no difference was identified for the relapse rate or limited cGVHD as reported elsewhere $(8,14)$. By contrast, a previous study by Ahmad et al (7) did not show improved OS in patients with cGVHD and eosinophilia in patients administered only reduced-intensity conditioning (RIC) with peripheral blood stem cell transplant as stem cell source for allogeneic recipients, of which the vast majority had myeloma or non-Hodgkin lymphoma (NHL) as the underlying disease. Similarly, a direct comparison between the results of Ahmad et al (7), the results of the present study and other studies $(8,14)$ is difficult due to the heterogeneity of the patients and transplant characteristics, such as conditioning intensity, stem cell source and underlying disease.

Conflicting results have not only been reported for eosinophilia and the overall outcome after allo-HSCT, but also for the correlation of eosinophilia with the development of cGVHD. A number of studies have shown no correlation of eosinophilia to cGVHD, whereas according to other studies eosinophilia precedes or parallels the development of cGVHD (9,12-14,16,17). Kim et al (14) reported a biphasic pattern in the course of eosinophilia following allo-HSCT with two peaks, one prior to day +100 and one $\sim 200$ days after HSCT, possibly explaining the conflicting results concerning eosinophilia and GVHD in previous studies.

As a result, the exact time course of eosinophil counts in cGVHD patients before and after diagnosis, taking into consideration steroid treatment, was investigated in this study. Two different patterns of the relative eosinophil course were identified. Patients exhibiting later increasing or decreasing RECs following diagnosis showed relatively stable counts from day -14 to day -7 , but showed a similar increase at the day of diagnosis. The other pattern was observed in patients without significant RECs changes. Eosinophil counts remained stable over time and did not show any increase at the day of the diagnosis most likely due to the higher administered steroid dose observed in this group. Previous studies in solid organ 


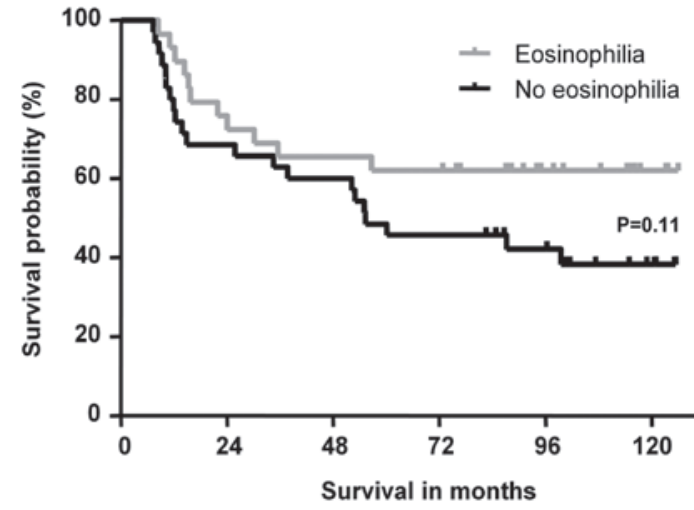

Figure 1. Kaplan-Meier curve of the 64 chronic graft-versus-bone disease (cGVHD) patients stratified for the presence of eosinophilia. The gray line represents the survival curve of cGVHD patients with eosinophilia $>4 \%$ ( $n=29$, $45 \%)$ and the black line represents patients without eosinophilia $(n=35,55 \%)$.
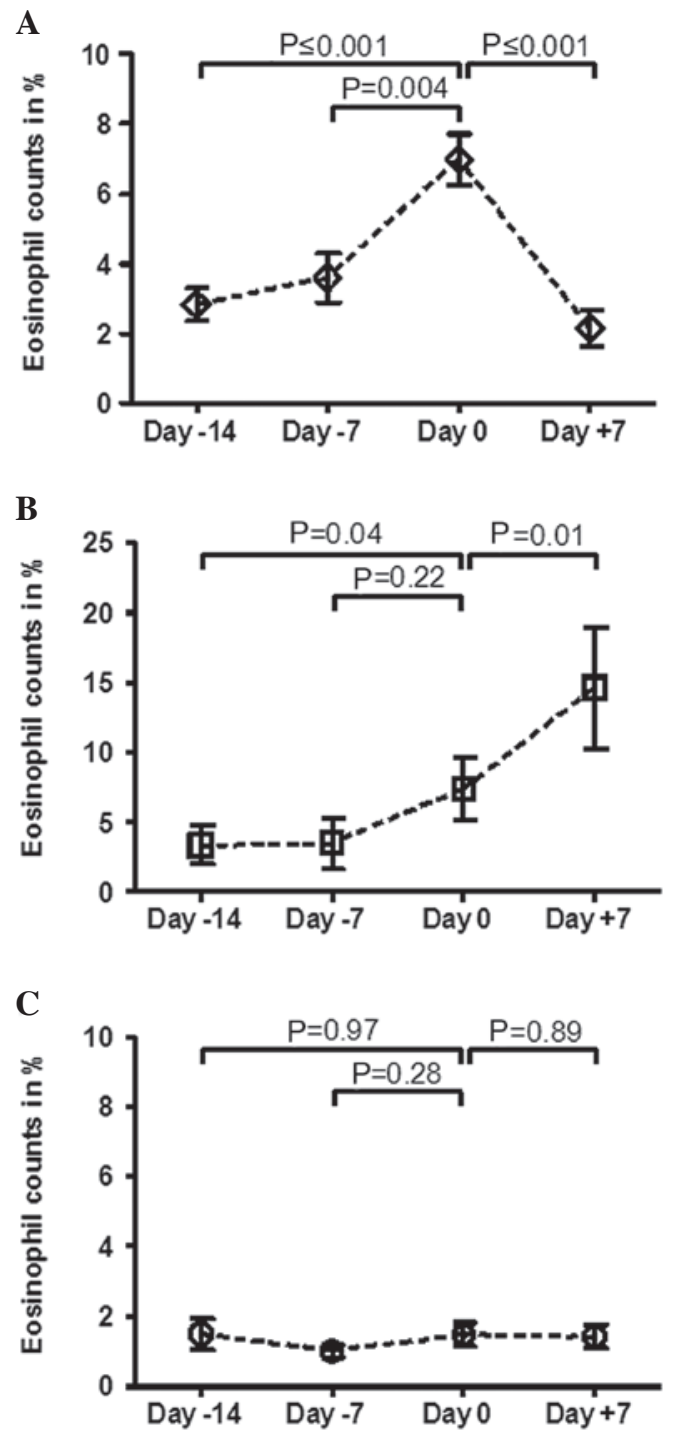

Figure 2. Course of peripheral blood eosinophils in chronic graft-versus-bone disease (cGVHD) patients. Peripheral blood eosinophil counts in relation to the first time point of cGVHD diagnosis are shown as mean RECs (\% of total leukocytes) \pm standard error of the mean (SEM) for days -14, -7, 0 (day of cGVHD diagnosis) and +7. (A) Eosinophil course of patients with decreasing eosinophil counts after cGVHD diagnosis. (B) Eosinophil course of patients with additional increasing eosinophil counts after cGVHD diagnosis. (C) Eosinophil course of patients without relevant changes of the eosinophil counts.

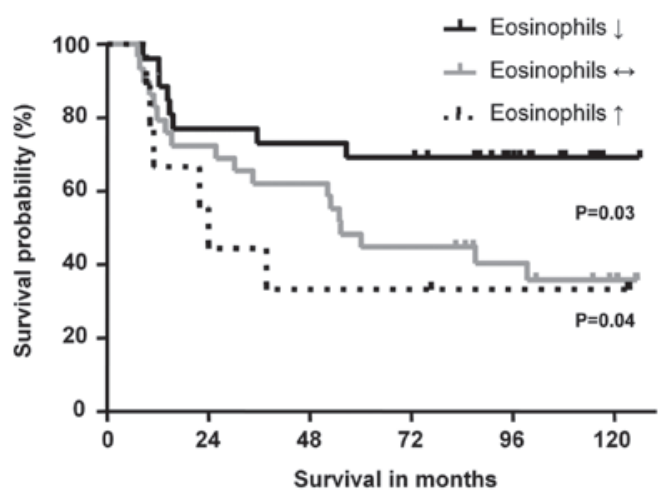

Figure 3. Kaplan-Meier curves for the changes of eosinophil counts after chronic graft-versus-bone disease (cGVHD) diagnosis in the three groups. The black line represents the patients with decreasing eosinophil counts after cGVHD diagnosis, the gray line patients without significant changes of the eosinophil counts, and the dashed black line patients with additional increasing eosinophil counts after cGVHD diagnosis.

transplantation have demonstrated that eosinophilia frequently occurs 2-4 days prior to the onset of graft rejection $(19,20)$. In agreement with the patient groups in the present study, Ahmad et al (7) reported an increase of the eosinophilia counts 4.5 days prior to diagnosis and a prompt decrease of the eosinophil counts after the initiation of cGVHD treatment.

However, not all patients with cGVHD developed eosinophilia with RECs $>4 \%$ or relevant changes of the eosinophil counts, explaining the potentially insufficient value of eosinophilia as a sensitive predictive marker for cGVHD diagnosis due to low sensitivity. However, a high specificity of eosinophilia for acute rejection in solid organ transplantation has been reported $(19,20)$.

Focusing on eosinophil counts as a marker for cGVHD activity including changes of subclinical RECs not being classified as eosinophilia, we investigated patients depending on the course of the eosinophil counts the week after diagnosis. A significant survival advantage of patients with decreasing RECs compared to patients with additional increasing RECs the week after diagnosis was identified. The two groups did not differ in terms of relapse rate and limited cGVHD, while patients with further increasing RECs exhibited a significantly increased rate of later cGVHD aggravation, possibly contributing to the impaired OS. In agreement with this observation, patients without significant RECs changes also exhibited a strong tendency to later cGVHD aggravation. Concerning additional factors affecting OS (16), the role of significant higher percentage of previous history of acute GVHD in the group with increasing RECs compared to other groups remains ambiguous since none of the patients exhibited cGVHD overlapping from acute GVHD. In addition, the multivariate analysis suggested only age and relapse as independent factors for survival.

Notably, during the analysis of steroid treatment as a possible bias on eosinophil counts, the percentage of patients administered steroid dosage escalation as part of the cGVHD therapy did not differ in the decreasing and increasing course of RECs groups. However, higher steroid doses already applied prior to cGVHD diagnosis could cover changes of eosinophil counts as observed in patients without significant RECs 
changes. In addition, the possibility that other factors apart from the supposed Th2 cytokine pattern inducing eosinophilia may contribute to cGVHD pathophysiology could not be excluded $(4,5)$.

In conclusion, the results of this study support the role of eosinophil counts changes as a marker for cGVHD activity and future severity. A further increase of RECs the week after cGVHD diagnosis indicates uncontrolled disease activity as observed in a higher percentage of later cGVHD aggravation and this may contribute to impaired OS. By contrast, spontaneous or possibly steroid-induced decreasing eosinophil counts account for better control of cGVHD disease activity, indicated by a reduced frequency of later cGVHD aggravation and, consequently, an improved OS. Due to the need for further improvement of therapy and treatment decision making in cGVHD patients, eosinophil decrease the week following diagnosis may help identify those patients that may benefit from graft versus leukemia effects in the presence of controllable cGVHD. For more detailed investigation, prospective clinical trials on larger patient cohorts with well-defined testing interventions directed to the pathophysiology of the disease, may help to identify additional groups of patients that could benefit from cGVHD and gain a better understanding of the correlation between eosinophils, Th2 cytokines, cGVHD and its treatment.

\section{Acknowledgements}

The authors would like to thank Christoph P. Beier for reviewing the manuscript.

\section{References}

1. Rothenberg ME: Eosinophilia. N Engl J Med 338: 1592-1600, 1998.

2. Akpek G, Zahurak ML, Piantadosi S, et al: Development of a prognostic model for grading chronic graft-versus-host disease. Blood 97: 1219-1226, 2001.

3. Filipovich AH, Weisdorf D, Pavletic S, et al: National Institutes of Health consensus development project on criteria for clinical trials in chronic graft-versus-host disease: I. Diagnosis and staging working group report. Biol Blood Marrow Transplant 11: 945-956, 2005.

4. Baird K and Pavletic SZ: Chronic graft versus host disease. Curr Opin Hematol 13: 426-435, 2006.
5. Ferrara JL, Levine JE, Reddy P and Holler E: Graft-versus-host disease. Lancet 373: 1550-1561, 2009.

6. Landfried K, Wolff D and Holler E: Pathophysiology and management of graft-versus-host disease in the era of reduced-intensity conditioning. Curr Opin Oncol 21 (Suppl 1): S39-S41, 2009.

7. Ahmad I, Labbe AC, Chagnon M, et al: Incidence and prognostic value of eosinophilia in chronic graft-versus-host disease after nonmyeloablative hematopoietic cell transplantation. Biol Blood Marrow Transplant 17: 1673-1678, 2011.

8. Aisa Y, Mori T, Nakazato T, et al: Blood eosinophilia as a marker of favorable outcome after allogeneic stem cell transplantation. Transpl Int 20: 761-770, 2007.

9. Arslan O, Akan $\mathrm{H}, \mathrm{Koc} \mathrm{H}$, et al: Eosinophilia after allogeneic bone marrow transplantation using busulfan and cyclophosphamide conditioning regimen. Bone Marrow Transplant 18: 261, 1996.

10. Basara N, Kiehl MG and Fauser AA: Eosinophilia indicates the evolution to acute graft-versus-host disease. Blood 100: 3055, 2002.

11. Imahashi N, Miyamura K, Seto A, et al: Eosinophilia predicts better overall survival after acute graft-versus-host-disease. Bone Marrow Transplant 45: 371-377, 2010.

12. Jacobsohn DA, Schechter T, Seshadri R, Thormann K, Duerst R and Kletzel M: Eosinophilia correlates with the presence or development of chronic graft-versus-host disease in children. Transplantation 77: 1096-1100, 2004.

13. Kalaycioglu ME and Bolwell BJ: Eosinophilia after allogeneic bone marrow transplantation using the busulfan and cyclophosphamide preparative regimen. Bone Marrow Transplant 14: 113-115, 1994.

14. Kim DH, Popradi G, Xu W, et al: Peripheral blood eosinophilia has a favorable prognostic impact on transplant outcomes after allogeneic peripheral blood stem cell transplantation. Biol Blood Marrow Transplant 15: 471-482, 2009.

15. McNeel D, Rubio MT, Damaj G, et al: Hypereosinophilia as a presenting sign of acute graft-versus-host disease after allogeneic bone marrow transplantation. Transplantation 74: 1797-1800, 2002.

16. Przepiorka D, Anderlini P, Saliba R, et al: Chronic graft-versushost disease after allogeneic blood stem cell transplantation. Blood 98: 1695-1700, 2001.

17. Sato T, Kobayashi R, Nakajima M, Iguchi A and Ariga T: Significance of eosinophilia after stem cell transplantation as a possible prognostic marker for favorable outcome. Bone Marrow Transplant 36: 985-991, 2005.

18. Alegre ML, Florquin S and Goldman M: Cellular mechanisms underlying acute graft rejection: time for reassessment. Curr Opin Immunol 19: 563-568, 2007.

19. Nagral A, Ben-Ari Z, Dhillon AP and Burroughs AK: Eosinophils in acute cellular rejection in liver allografts. Liver Transpl Surg 4: 355-362, 1998.

20. Foster PF, Sankary HN, Hart M, Ashmann M and Williams JW: Blood and graft eosinophilia as predictors of rejection in human liver transplantation. Transplantation 47: 72-74, 1989. 